Journal of Low Temperature Physics manuscript No.

(will be inserted by the editor)

R. Gualtieri ${ }^{1}$ - J.P. Filippini ${ }^{1,2}$. P.A.R. Ade $^{3}$ - M. Amiri ${ }^{4}$. S.J. Benton ${ }^{5}$.

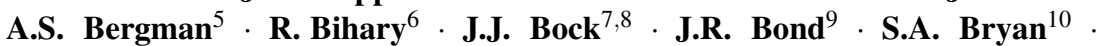

H.C. Chiang ${ }^{11,12}$. C.R. Contaldi ${ }^{13}$. O. Doré C.,8 $^{7}$ A.J. Duivenvoorden ${ }^{14}$.

H.K. Eriksen ${ }^{15}$. M. Farhang ${ }^{9,16}$. L.M. Fissel ${ }^{16,17}$ - A.A. Fraisse $^{5}$ - K.

Freese $^{14,18}$ - M. Galloway ${ }^{19}$ - A.E. Gambrel ${ }^{5}$ - N.N. Gandilo ${ }^{20,21}$. K. Ganga ${ }^{22}$.

R.V. Gramillano ${ }^{1} \cdot$ J.E. Gudmundsson ${ }^{14} \cdot$ M. Halpern ${ }^{4} \cdot$ J. Hartley $^{19} \cdot$ M.

Hasselfield $^{23}$. G. Hilton ${ }^{24}$. W. Holmes ${ }^{8}$. V.V. Hristov ${ }^{7}$. Z. Huang ${ }^{9}$. K.D.

Irwin $^{25,26}$. W.C. Jones ${ }^{5}$. C.L. Kuo ${ }^{25}$. Z.D. Kermish ${ }^{5}$. S. Li $\mathbf{L i}^{5,16,27}$. P.V.

Mason $^{7}$. K. Megerian ${ }^{8}$. L. Moncelsi ${ }^{7}$. T.A. Morford ${ }^{7}$. J.M. Nagy ${ }^{28,6}$. C.B.

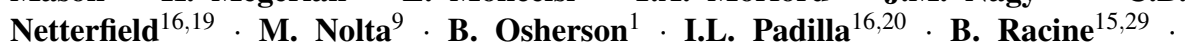

A.S. Rahlin $^{30,31}$. C. Reintsema ${ }^{24}$ - J.E. Ruhl ${ }^{6}$ - M.C. Runyan ${ }^{8}$. T.M. Ruud ${ }^{15}$.

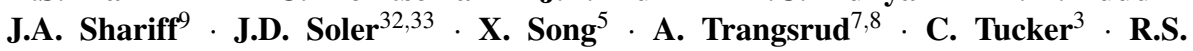
Tucker $^{7}$ - A.D. Turner ${ }^{8}$. J.F. van der List ${ }^{5}$ - A.C. Weber ${ }^{8}$ - I.K. Wehus ${ }^{15}$. D.V. Wiebe $^{4}$. E.Y. Young ${ }^{5}$

\title{
SPIDER: CMB polarimetry from the edge of space
}

the date of receipt and acceptance should be inserted later

\footnotetext{
${ }^{1}$ Department of Physics, University of Illinois at Urbana-Champaign, Urbana, IL, USA

${ }^{2}$ Department of Astronomy, University of Illinois at Urbana-Champaign, Urbana, IL, USA

${ }^{3}$ School of Physics and Astronomy, Cardiff University, CF24 3AA, UK

${ }^{4}$ Department of Physics and Astronomy, University of British Columbia, Vancouver, BC, Canada

${ }^{5}$ Department of Physics, Princeton University, Princeton, NJ, USA 08544, USA

${ }^{6}$ Physics Department, Center for Education and Research in Cosmology and Astrophysics, CaseWestern Reserve University, Cleveland, OH, USA

${ }^{7}$ Division of Physics, Mathematics and Astronomy, California Institute of Technology, Pasadena, CA, USA

${ }^{8}$ Jet Propulsion Laboratory, Pasadena, CA, USA

${ }^{9}$ Canadian Institute for Theoretical Astrophysics, University of Toronto, Toronto, ON, Canada

${ }^{10}$ School of Earth and Space Exploration, Arizona State University, Tempe, AZ, USA

${ }^{11}$ School of Mathematics, Statistics and Computer Science, University of KwaZulu-Natal, Durban, South Africa

${ }^{12}$ National Institute for Theoretical Physics (NITheP), KwaZulu-Natal, South Africa

${ }^{13}$ Blackett Laboratory, Imperial College London, London, UK

${ }^{14}$ The Oskar Klein Centre for Cosmoparticle Physics, Department of Physics, Stockholm University, Stockholm, Sweden

${ }^{15}$ Institute of Theoretical Astrophysics, University of Oslo, Oslo, Norway

${ }^{16}$ Department of Astronomy and Astrophysics, University of Toronto, Toronto, ON, Canada

${ }^{17}$ National Radio Astronomy Observatory, Charlottesville, NC, USA

${ }^{18}$ Department of Physics, University of Michigan, Ann Arbor, MI, USA

${ }^{19}$ Department of Physics, University of Toronto, Toronto, ON, Canada

${ }^{20}$ Department of Physics and Astronomy, Johns Hopkins University, Baltimore, MD, USA

${ }^{21}$ NASA Goddard Space Flight Center, Greenbelt, MD, USA

${ }^{22}$ APC, Univ. Paris Diderot, CNRS/IN2P3, CEA/Irfu, Obs de Paris, Sorbonne Paris Cite, France

${ }^{23}$ Pennsylvania State University, University Park, PA, USA

${ }^{24}$ National Institute of Standards and Technology, Boulder, CO, USA

${ }^{25}$ Department of Physics, Stanford University, Stanford, CA, USA

${ }^{26}$ SLAC National Accelerator Laboratory, Menlo Park, CA, USA

${ }^{27}$ Department of Mechanical and Aerospace Engineering, Princeton University, Princeton, NJ, USA

${ }^{28}$ Dunlap Institute for Astronomy \& Astrophysics, University of Toronto, Toronto, ON, Canada

${ }^{29}$ Harvard-Smithsonian Center for Astrophysics, Cambridge, MA, USA

${ }^{30}$ Fermi National Accelerator Laboratory, Batavia, IL, USA

${ }^{31}$ Kavli Institute for Cosmological Physics, University of Chicago, Chicago, IL, USA

${ }^{32}$ Max-Planck-Institute for Astronomy, Heidelberg, Germany

${ }^{33}$ Laboratoire AIM, Paris-Saclay, CEA/IRFU/Sap-CNRS-Université Paris Diderot, Gif-sur-Yvette Cedex,

France E-mail: rgualtie@illinois.edu
} 


\begin{abstract}
SPIDER is a balloon-borne instrument designed to map the polarization of the millimeter-wave sky at large angular scales. SPIDER targets the $B$-mode signature of primordial gravitational waves in the cosmic microwave background (CMB), with a focus on mapping a large sky area with high fidelity at multiple frequencies. SPIDER's first longduration balloon (LDB) flight in January 2015 deployed a total of 2400 antenna-coupled Transition Edge Sensors (TESs) at $90 \mathrm{GHz}$ and $150 \mathrm{GHz}$. In this work we review the design and in-flight performance of the SPIDER instrument, with a particular focus on the measured performance of the detectors and instrument in a space-like loading and radiation environment. SPIDER's second flight in December 2018 will incorporate payload upgrades and new receivers to map the sky at $285 \mathrm{GHz}$, providing valuable information for cleaning polarized dust emission from CMB maps.
\end{abstract}

Keywords Cosmic microwave background, inflation, bolometers, transition edge sensors, polarimetry

\title{
1 Introduction
}

Precision observations of the CMB have transformed our understanding of the universe's history and composition. The next frontier of this endeavor is the characterization of the exceedingly faint pattern of polarization in this radiation field, with an intensity more than an order of magnitude smaller than that of the CMB's well-known temperature anisotropies. An accurate map of this polarization across the sky encodes information about the local conditions within the primordial plasma that is complementary to that available from the temperature. Most intriguingly, the presence of an odd-parity " $B$-mode" pattern of polarization at large angular scales would be an unambiguous signature that our universe began with an inflationary epoch of rapid expansion, thus providing information on fundamental physics at energy scales far beyond those achievable at accelerators $\frac{1}{1}$. A believable detection (or exclusion) of this faint signal will require polarimeters of enormous sensitivity, exquisite control of polarized instrumental systematics, and clean separation of the CMB from galactic and atmospheric foregrounds. Numerous scientific teams are now developing and deploying terrestrial, balloon-borne, and satellite instruments to seek out this signature in the millimeter-wave sky. Current observational constraints limit the tensor-to-scalar ratio $r$ - the power of primordial gravitational waves relative to that of the primordial density perturbations - to be less than 0.07 at $95 \%$ confidence ${ }^{213}$.

SPIDER $^{4} 6$ is a balloon-borne telescope array optimized to search for the signature of cosmic inflation ${ }^{7}$ in the polarization of the $\mathrm{CMB}$, and to discriminate a primordial signal from galactic foreground emission. SPIDER employs multiplexed arrays of transition-edge sensor (TES) bolometers to observe the millimeter-wave sky from a NASA LDB. LDB flights from McMurdo Station, Antarctica, provide multi-week flights at high $(\sim 36 \mathrm{~km})$ observing altitudes, near-space photon backgrounds, and sensitivity to frequencies and angular scales that are contaminated by atmosphere at even the most favorable ground-based observing sites. In addition to their observing advantages, LDB flights are also technological

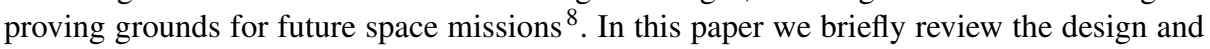
performance of the SPIDER instrument, with a particular focus on SPIDER's performance in this environment during its first observing flight in January 2015. 

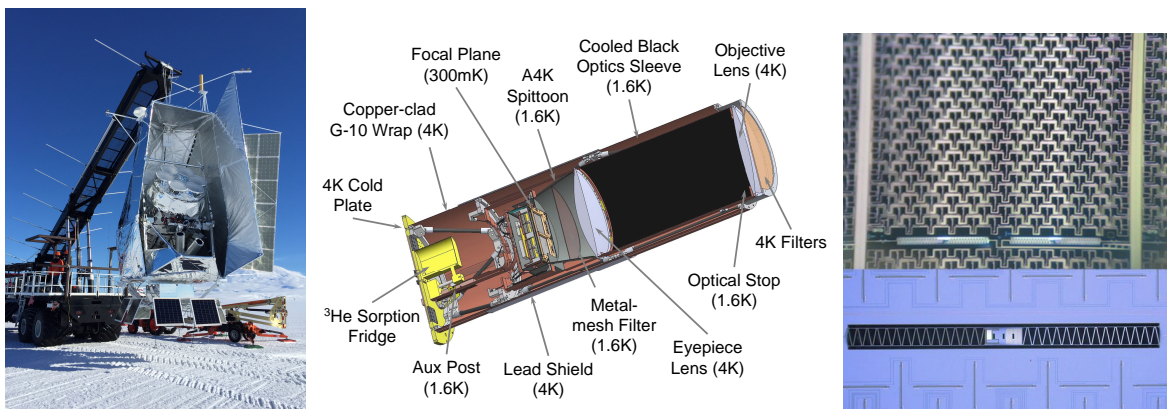

Fig. 1: Left: Photograph of the complete SPIDER payload hanging from the LDB launch vehicle. The six telescope aperture forebaffles are visible emerging from the central cryostat, which is supported by a scanning carbon fiber gondola. A sun shield surrounds the assembly, and a solar panel "wing" is visible at right. Center: Cross-section of the SPIDER telescope model with key components labeled. Top right: Antenna-coupled TES pixel as used in the first flight, showing phased array of slot antennas feeding two TESs at bottom. Bottom right: Close-up of a single TES assembly, showing the TES island and the SiN thermal isolation legs. The meandered leg design allows for low thermal conductance in a narrow geometry.

\section{The SPIDER 2015 payload}

\subsection{Overview}

The SPIDER payload (Fig. [1 left) consists of six monochromatic refracting telescopes housed within a single large liquid helium cryostat. The cryostat and associated electronics are supported within a lightweight carbon fiber gondola, which hangs beneath the balloon ${ }^{9}$.

A reaction wheel and linear drive scan the cryostat in azimuth and elevation throughout the flight working in concert with a motorized pivot, at speeds as high as $4^{\circ} / \mathrm{s}$ in azimuth 10 . A suite of star cameras, GPS receivers, sun sensors, and gyroscopes enable accurate instantaneous $\left(<5^{\prime} \mathrm{rms}\right)$ and post-flight $\left(<5^{\prime \prime} \mathrm{rms}\right)$ pointing reconstruction 11 , well in excess of requirements for the relatively broad beams $\left(30^{\prime}\right.$ FWHM at $150 \mathrm{GHz}$ and $42^{\prime}$ FWHM at $90 \mathrm{GHz}$ ) of the SPIDER telescopes. A set of sun shields protects the instrument and optics during the 24-hour Antarctic summer daylight. A $2 \mathrm{~kW}$ solar panel system provides electric power throughout the flight. An assortment of antenna arrays provide commanding, telemetry, and location information during flight.

SPIDER's cryogenic system, the largest yet deployed on an LDB, consists of two liquid helium reservoirs: a $1284 \mathrm{~L}$ main tank and a $20 \mathrm{~L}$ superfluid tank ${ }^{12}$. The main tank is maintained near sea level atmospheric pressure during the flight, providing each telescope with cooling power at $\sim 4 \mathrm{~K}$. Vapor from the main tank cools a series of vapor-cooled shields to reduce radiative load on the cryogenic system. The superfluid tank fills continuously from the main tank through a capillary assembly, and is pumped out to about 5 torr by opening a valve to the low pressure atmosphere at float. The superfluid system provides cooling power at $1.6 \mathrm{~K}$ to each inserts' sub-Kelvin cooler and internal optical baffles. The focal planes themselves are cooled to $\sim 300 \mathrm{mK}$ by a dedicated ${ }^{3} \mathrm{He}$ adsorption refrigerator within each telescope. 


\subsection{Receivers}

Each SPIDER receiver is a two-lens cold refracting telescopes, adapted from the basic plan of the BICEP 1 instrument ${ }^{13 / 14}$. The SPIDER lens design is optimized for a planar-antenna focal plane, while the filter stack and telescope structure incorporate several adaptations for a balloon-borne environment. Each telescope consists of two anti-reflection coated polyethylene lenses cooled to $4 \mathrm{~K}$, focusing light onto the focal plane (Fig. 1. center). This compact, axisymmetric design limits polarized systematics within the instrument. The relatively small $(26 \mathrm{~cm} \varnothing)$ stop aperture simplifies baffling and far-field beam characterization while retaining sufficient angular resolution to map the inflationary $B$-mode signal expected at degree angular scales. This modular design using monochromatic telescopes greatly simplifies antireflection coatings for the optical elements and allows for flexibility in the instrument's frequency coverage. Each receiver is supported by a rigid carbon fiber frame to reduce payload mass, a critical concern for balloon-borne operations.

A primary concern for the SPIDER optical system has been to reduce stray optical load on the detectors, which degrades mapping speed and would eventually saturate the TES bolometers. This has forced several adaptations from the BICEP2 design?. A blackened "sleeve" baffle cooled to $\lesssim 2$ Kelvin by the superfluid tank has been added between the secondary lens and the Lyot stop to reduce loading from the telescope itself. SPIDER's filter stack is predominantly reflective (metal-mesh filters ${ }^{16}$ ) to further reduce emission, with a single absorptive nylon filter at $4 \mathrm{~K}$. The vacuum seal for each telescope is provided by a thin sheet of anti-reflection coated ultra-high molecular weight polyethylene, with low scattering and emissivity. The warm baffling of each telescope is primarily reflective rather than absorptive, redirecting side lobe response to the cold sky rather than absorbing it at ambient temperature.

Polarization modulation for each telescope is provided by a sapphire half-wave plate ${ }^{17 \mid 18}$, just skyward of the 4 Kelvin filter stack and also cooled to $4 \mathrm{~K}$. Each plate's thickness and coating are tuned for the wavelength of the associated receiver, yielding minimal impact on optical throughput, ghosting, and pointing. Each wave plate is operated in a stepped configuration, rotated every $\sim 12$ hours during fridge cycling to provide polarization modulation over the course of the flight.

\subsection{Focal plane assemblies}

In SPIDER's 2015 flight, these optical systems focused light onto planar arrays of dualpolarization antenna-coupled TESs developed at Caltech/JPL 19. Each receiver's focal plane houses four silicon tiles, each photolithographically patterned with an $8 \times 8$ square array $(6 \times 6$ for $90 \mathrm{GHz})$ of polarimeter pixels. Each of these consists of two interpenetrating arrays of slot antennas sensitive to perpendicular polarization modes (Figure 1 top right). The power received by each beam-forming antenna array is fed to a dedicated Ti TES $\left(T_{c} \sim 500\right.$ $\mathrm{mK}$ ) through an inline band-defining filter. The arrays are read out using a time-division Superconducting QUantum Interference Device (SQUID) multiplexer system²0|21. A second $\mathrm{Al}$ TES $\left(T_{c} \sim 1.3 \mathrm{~K}\right)$ is wired in series with the primary Ti TES to allow receiver characterization under laboratory loading conditions.

SPIDER's detectors are optimized to take advantage of the extremely low optical loading from the sky at balloon altitudes, $<1 / 10$ that available at the South Pole. SPIDER TESs use a meandered leg design (Fig. 1 bottom right) that gives lower thermal conductance (and thus lower thermodynamic noise) in a similar footprint area. Typical thermal conductances 
of the completed SPIDER arrays are of order $G \sim 11-20 \mathrm{pW} / \mathrm{K}$ at $450 \mathrm{mK}$. This solution is different in example from the straight-legged design used for the isolation membranes of the BICEP2 bolometers 22 . The electrical noise of the amplifier is kept subdominant to that from the detectors and photons by reducing the detector resistance and increasing the readout circuit inductance.

The focal plane housing the detectors and SQUIDs has been designed from the ground up to provide excellent shielding against time-varying magnetic fields, using several nested layers of superconducting and ferromagnetic materials. Without such shielding the payload's motion in the Earth's magnetic field could induce spurious signals into the readout system. This is a greater concern for SPIDER than for terrestrial instruments, since the gondola's motion makes it difficult to subtract such effects with a ground-fixed template. For further details of this design, see Runyan et al. $2010^{23}$.

\section{In-flight performance}

\subsection{5 flight}

SPIDER took to the air from the LDB base camp at McMurdo Station, Antarctica on January 1, 2015. All systems functioned well during the 16-day flight, with the exception of a redundant differential GPS package. The flight terminated successfully in West Antarctica, $2270 \mathrm{~km}$ from the launch point. SPIDER's data disks were recovered in February 2015, and the full payload in November 2015, by the British Antarctic Survey. All hardware has been returned to North America, and is currently being refit for SPIDER's upcoming second flight (see Section 4).

Robust automation was a key consideration for SPIDER's flight operations. The limited satellite link bandwidth prohibited a live downlink of SPIDER's full data set, necessitating on-board data quality checks and error recovery. Payload telemetry was also limited, due both to bandwidth demands and to radio-frequency interference (RFI) induced in the detectors by the onboard higher-bandwidth antennas. Instead, the redundant flight computer systems monitored payload and detector status onboard, adjusting detector biases and other settings as needed. Highly compressed diagnostic packets were transmitted to the ground station in brief bursts at regular intervals, limiting the impact on data. The higher bandwidth satellite links were reserved for on-demand use to examine specific issues, generally during cryogenic servicing operations when science data were not being acquired.

During its flight SPIDER mapped approximately $10 \%$ of the sky at 90 and $150 \mathrm{GHz}$, using three receivers at each frequency. The resulting $\sim 1.6 \mathrm{~TB}$ of data are currently under analysis 24 .

\subsection{Detector performance}

The 2015 flight incorporated a total of 2400 TES bolometers, of which 96 were intentionally not coupled to antennas. After accounting for hardware yield and relatively stringent data selection cuts, we currently retain data from 1863 optically-coupled bolometers $(675 / 1188$ at $90 / 150 \mathrm{GHz})$. We further exclude periods of data on individual detectors for various causes, most prominently RFI from the communications arrays. The estimated noise-equivalent temperature (NET) of the total instrument after these cuts is approximately $7.1(5.3) \mu \mathrm{K}_{c m b} \sqrt{s}$ at 90 (150) GHz. 
Due to SPIDER's cold optical chain, radiation loading from the instrument itself was kept extremely low. We estimate a total in-band loading during flight of $\lesssim 0.25(\lesssim 0.35) \mathrm{pW}$ at 90 (150) GHz. This includes loading from the instrument, the atmosphere, and the CMB itself. Those numbers are comparable to, or lower than, those achieved by Planck-HFI at L2 25 , a significant technical achievement and benefit of the LDB platform. If outfitted with truly photon-noise-limited detectors, a similar design might thus quadruple SPIDER's present mapping speed.

\subsection{Cosmic ray response}

One important challenge of a space or near-space environment is the greatly increased flux of particle radiation relative to that at sea level. The near-Earth environment is pelted by radiation of solar, galactic, and extra-galactic origins, predominantly a broad spectrum of protons peaking near $300 \mathrm{MeV}$ and extending well beyond the $\mathrm{TeV}$ range. Frequent glitches in the data stream from particle interactions have been a source of analysis difficulty for the Planck satellite $\frac{26}{}$, and will be a factor in the design of future space-based bolometric instruments. This particle flux is lower at balloon altitudes, but is still an important part of SPIDER's data analysis. Below we give a first description of the general features of cosmic ray-induced glitches seen in the SPIDER data set.

Particle events deposit energy in the TES bolometer, producing brief glitches in the data that generally recover quickly according the bolometer's (optical) time constant. The typical energy deposition in a component is determined predominantly by its thickness, while the glitch rate is determined by its area. The incident particle rate is affected by solar activity, surrounding materials, and (for a balloon) the Earth's magnetic field. From a highly simplified model, we expect energy depositions of order $\sim 1 \mathrm{keV}$ in each bolometer every few minutes, while the larger wafer experiences MeV-scale depositions at $\sim 100 \mathrm{~Hz}$. The rate of bolometer depositions should thus be manageable in analysis, as long as the TES recovers quickly from each glitch. Of more concern are response to bolometer hits in the wafer and multiplexer crosstalk, both of which could greatly increase the data loss.

Cosmic ray-induced glitches (CRGs) are seen in SPIDER 2015 data every $\sim 3$ minutes in each detector. Typical glitches are only a handful of samples in length at an acquisition rate of $126 \mathrm{~Hz}$, with shape determined by the digital anti-aliasing filter applied by our readout electronics. These glitches are flagged and removed with minimal effect on our science analysis. Laboratory characterization of an antenna-coupled TES array with a ${ }^{32} \mathrm{~S}$ source at Caltech revealed that the SQUID readout system could occasionally lose lock during a large energy deposition, causing the DC level of the data stream to be offset by one flux quantum. Such "flux-slip" discontinuities are seen regularly in SPIDER data, primarily due to RFI but occasionally due to energetic particle interactions. They are corrected during low-level data processing.

Fig. 2 shows the estimated spectrum of deposited energies for cosmic ray glitches in a representative focal plane unit (FPU). Glitch rates are 10-100 $\times$ lower than in Planck $\mathrm{HFI}^{28}$, qualitatively consistent with the somewhat more benign environment in the stratosphere. Coincidence rates are low $(\sim 0.03 \%)$, suggesting relatively little long-distance energy propagation through the silicon tile. No long "tails" are apparent in the pulse decay times, but this is difficult to constrain given the digital filter. Laboratory tests with radioactive sources are in progress, and will give further information on this front. 

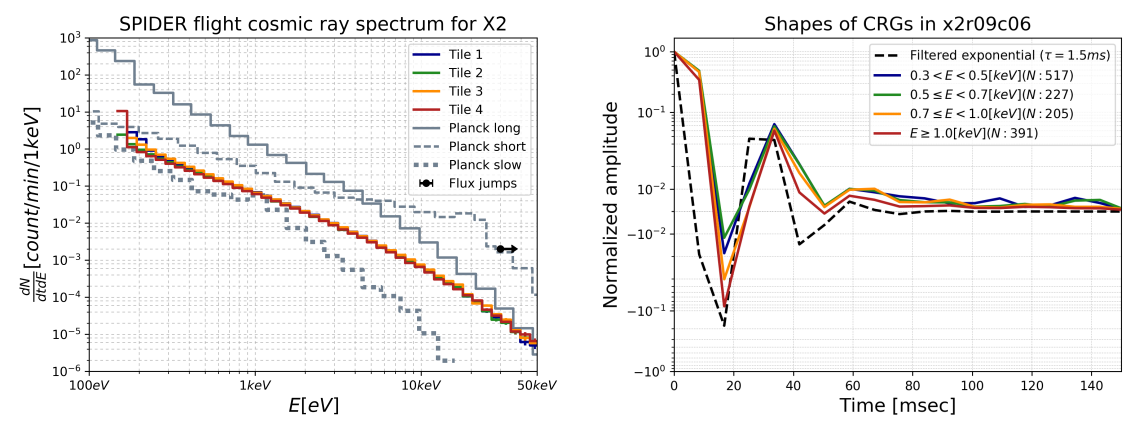

Fig. 2: Left: Estimated spectrum of deposited energy for cosmic ray glitches in each of the four silicon tiles for a single SPIDER receiver. Approximate energies are estimated from the integrated reduction in Joule heating of the TES. Also plotted is the rate of flux slip discontinuities seen by this FPU (black marker near $30 \mathrm{keV}$ ) and spectra for the three types of CRG events seen in Planck ${ }^{28}$. Right: Averaged traces of CRGs seen in one representative detector. The pulse shape is relatively independent of energy, and largely established by the digital anti-aliasing filter.

\section{Status and prospects}

SPIDER's first scientific result was published in 2017: a new constraint on the level of circular polarization in the CMB and millimeter-wave sky (Fig. 3. Left). This analysis takes advantage of non-idealities in SPIDER's half-wave plates that couple circular polarization into linear polarization, leading to constraints three orders of magnitude stronger than previous work $^{24}$. SPIDER's primary B-mode polarization analysis is ongoing.
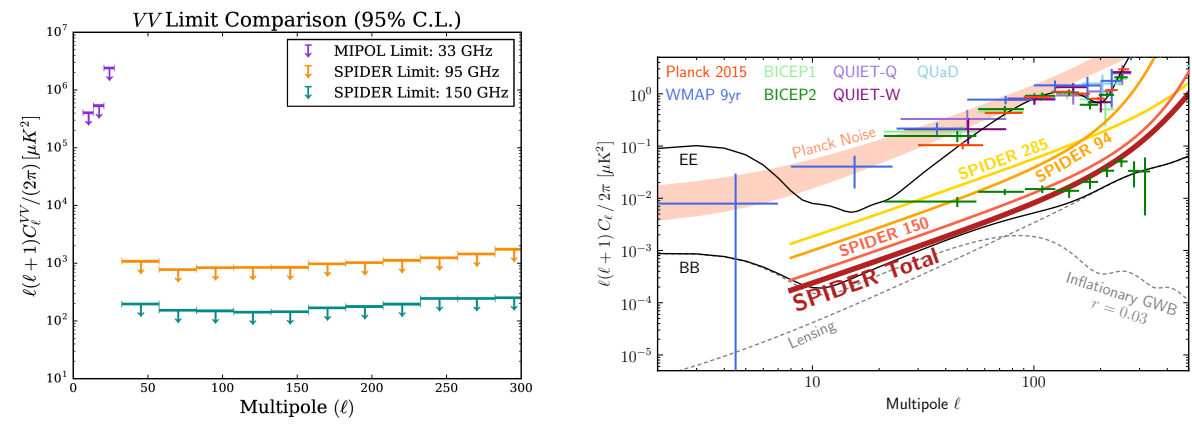

Fig. 3: Left: Upper limits on circular polarization sky power at 90 and $150 \mathrm{GHz}$ as a function of multipole, including comparison to previous results. Figure from Nagy et al. (2017) 24 . Right: Forecast BB sensitivity as a function of multipole for SPIDER after two flights, in comparison with some other recent experiments.

The SPIDER collaboration is preparing for a second observing flight in Dec. 2018, also on an Antarctic LDB. This flight will incorporate three new receivers to map the same sky at $280 \mathrm{GHz}$, using feedhorn-coupled arrays of AlMn TES polarimeters developed at NIST 29130. The resulting $280 \mathrm{GHz}$ data will yield maps of galactic dust polarization over a large sky fraction at a frequency in between the existing Planck sky maps, which will have lasting value in the field. SPIDER's second flight will also incorporate significant upgrades 
to the cryostat and gondola hardware. We expect SPIDER to be able to detect or constrain primordial gravitational waves at the $r>0.03$ at $3 \sigma$ confidence level (Fig. 3, Right). The results obtained in this work were possible thanks to the unique opportunity to study the CR environment from the stratosphere, for the first time with TESs, given by the data collected during the first successful SPIDER campaign. The analysis presented here will help the design and deployment of this detector's technology on future satellite mission.

Acknowledgements SPIDER is supported in the U.S. by the National Aeronautics and Space Administration under grants NNX07AL64G, NNX12AE95G, and NNX17AC55G issued through the Science Mission Directorate and by the National Science Foundation through PLR-1043515. Logistical support for the Antarctic deployment and operations was provided by the NSF through the U.S. Antarctic Program. Cosmic ray response studies are supported by NASA under grant 14-SAT14-0009. Support in Canada is provided by the National Sciences and Engineering Council and the Canadian Space Agency. Support in Norway is provided by the Research Council of Norway. Support in Sweden is provided by the Swedish Research Council through the Oskar Klein Centre (contract no. 638-2013-8993). KF acknowledges support from DoE grant de-sc0007859 at the University of Michigan. The collaboration is grateful to the British Antarctic Survey, particularly Sam Burrell, for invaluable assistance with data and payload recovery after the 2015 flight. We also wish to acknowledge the generous support of the David and Lucile Packard Foundation, which has been crucial to the success of the project. 


\section{References}

1. Dodelson, S., Easther, R., Hanany, S., et al., Astro2010: The Astronomy and Astrophysics Decadal Survey

2. BICEP2/Keck Collaboration and Planck Collaboration, Ade, P.A.R., Aghanim, N., Ahmed, Z. et al., Physical Review Letters, 114, 10, (2015), DOI: 10.1103/PhysRevLett.114.101301

3. Planck Collaboration, Ade, P.A.R., Aghanim, N., Arnaud, M. et al., Astronomy \& Astrophysics, , 594 , 65, (2016), DOI: 10.1051/0004-6361/201525898

4. Filippini, J. P., Ade, P. A. R., Amiri, M., et al., Proc. SPIE, 7741, (2010), DOI: $10.1117 / 12.857720$

5. Fraisse, A. A., Ade, P. A. R., Amiri, M., et al., Journal of Cosmology and Astroparticle Physics, 4, 047 (2013)

6. Rahlin, A. S., Ade, P. A. R., Amiri, M., et al., Proc. SPIE, 9153, (2014), DOI: $10.1117 / 12.2055683$

7. Guth, A. H., PRD, 23, 347-356, (1981), DOI: 10.1103/PhysRevD.23.347

8. Bock, J., Aljabri, A., Amblard, A., et al., arXiv:0906.1188 (2009)

9. Soler, J.D., Ade, P.A.R., Amiri, M. et al., Proc. SPIE, 9145, (2014), DOI: $10.1117 / 12.2055413$

10. Shariff, J. A., Ade, P. A. R., Amiri, M., et al., Proc. SPIE, 9145, (2014), DOI: 10.1117/12.2055166

11. Gandilo, N. N., Ade, P. A. R., Amiri, M., et al., Proc. SPIE, 9145, (2014), DOI: $10.1117 / 12.2055156$

12. Gudmundsson, J.E., Ade, P.A.R., Amiri, M. et al., Cryogenics, 72, 65-76, (2015), DOI: 10.1016/j.cryogenics.2015.09.002

13. Yoon, K. W., Ade, P. A. R., Barkats, D. et al., Proc. SPIE, 6275, (2006), DOI: $10.1117 / 12.672652$

14. Keating, B. G., Ade, P.A.R., Bock, J.J. et al., Proc. SPIE, 4843, 284-295, (2003), DOI: 10.1117/12.459274

15. BICEP2 Collaboration, Ade, P. A. R., Aikin, R. W., et al., Astrophys. J, 792, 62 (2014), DOI: 10.1088/0004-637X/792/1/62

16. Ade, P. A. R., Pisano, G., Tucker, C., \& Weaver, S., Proc. SPIE, 6275 (2006), DOI: 10.1117/12.673162

17. Bryan, S., Ade, P., Amiri, M. et al., Review of Scientific Instruments, 87, (2016), DOI: 10.1063/1.4939435

18. Bryan, S. A., Ade, P. A. R., Amiri, M., et al., Proc. SPIE, 7741, (2010), DOI: 10.1117/12.857837

19. BICEP2 Collaboration and Keck Array Collaboration and SPIDER Collaboration, Ade, P.A.R., Aikin, R.W., Amiri, M., The Astrophysical Journal, 812, 176, pp. 17, (2015), DOI: 10.1088/0004-637X/812/2/176

20. Battistelli, E. S., Amiri, M., Burger, B. et al., Journal of Low Temperature Physics, 151, 3, pp. 908-914, (2008), DOI: 10.1007/s10909-008-9772-Z

21. de Korte, P. A. J., Beyer, J., Deiker, S., et al., Review of Scientific Instruments, 74, 3807 (2003), DOI: 10.1063/1.1593809

22. BICEP2 Collaboration; Keck Array Collaboration; SPIDER Collaboration, Ade, P.A.R., Aikin, R.W., Amiri, M. et al, The Astrophysical Journal, 812, 17, (2015), DOI: 10.1088/0004-637X/812/2/176

23. Runyan, M.C., Ade, P.A.R., Amiri, M. et al., Proc. SPIE, 7741, (2010), DOI: $10.1117 / 12.857715$ 
24. Nagy, J. M., Ade, P. A. R., Amiri, M., et al., Astrophys. J, 844, 151 (2017), DOI: $10.3847 / 1538-4357 / \mathrm{aa} 7 \mathrm{cfd}$

25. Lamarre, J. M., Puget, J. L., Ade, P.A.R., Astronomy \& Astrophysics, 520, 20 (2010), DOI: $10.1051 / 0004-6361 / 200912975$

26. Planck Collaboration, Ade, P.A.R., Aghanim, N., Arnaud, M. et al., Astronomy \& Astrophysics, , 571 , A10, (2014), DOI: 10.1051/0004-6361/201321577

27. Perinati, E., Mineo, T., Colasanti, L. et al., Proc. SPIE, 7732, (2010), DOI: $10.1117 / 12.856393$

28. Catalano, A., Ade, P. and Atik, Y. et al., Journal of Low Temperature Physics, 176, 5, pp. 773-786, (2014), DOI: 10.1007/s10909-014-1116-6

29. Hubmayr, J., Austermann, J.E., Beall, J.A. et al., Proc. SPIE, 9914, (2016), DOI: $10.1117 / 12.2231896$

30. Bergman, A.S., Ade P.A.R., Akers, S. et al., Journal of Low Temperature Physics, This Special Issue (2017) 